\title{
Sodium-glucose co-transporter 2 inhibitors (SGLT2i); as a preventive factor of kidney failure in patients with type 2 diabetes; a meta-analysis of randomized controlled trials
}

\author{
Dorsa Jahangiri ${ }^{1,2}{ }^{\circledR}$, Udit Narayan Padhi ${ }^{{ }^{\circledR}}$, Henu Kumar Verma ${ }^{4}$, Bhaskar VKS Lakkakula ${ }^{3}$, Rohollah \\ Valizadeh $^{5,6 \mathbb{D}}$, Hamid Nasri ${ }^{*}$ (D) \\ ${ }^{1}$ Department of Internal Medicine, Isfahan University of Medical Sciences, Isfahan, Iran \\ ${ }^{2}$ Independent Researcher, 43185 Cardston Place Leesburg Virginia, 20176, USA \\ ${ }^{3}$ Department of Zoology, Guru Ghasidas Vishwavidyalaya, Bilaspur, India \\ ${ }^{4}$ Department of Immunopathology, Institute of lungs Biology and Disease, Comprehensive Pneumology Center, Helmholtz Zentrum, 85764 \\ Neuherberg, Munich, Germany \\ ${ }^{5}$ Student Research Committee, Department of Epidemiology, School of Public Health, Iran University of Medical Sciences, Tehran, Iran \\ ${ }^{6}$ Minimally Invasive Surgery Research Center, Hazrat-e Rasool General Hospital, Iran University of Medical Sciences, Tehran, Iran
}

\section{A R T I C L E I N F O}

\section{Article Type:}

Review

\section{Article History:}

Received: 3 June 2021

Accepted: 23 August 2021

Published online: 9 September 2021

\section{Keywords:}

Sodium-glucose transporter 2 inhibitors, Chronic kidney disease, Type 2 diabetes mellitus, Acute kidney injury, Meta-analysis

\begin{abstract}
A B S T R A C T
Introduction: Sodium-glucose co-transporter 2 inhibitors (SGLT2i) are a new class of antidiabetic drugs. SGLT2 inhibitors lower blood glucose levels by decreasing glucose reabsorption in the proximal renal tubule, resulting in increased urinary glucose and sodium excretion. Objective: This study was conducted to investigate the effects of SGLT2i on individual renal outcomes in diabetic patients.

Methods: This study was a systematic review and meta-analysis of clinical trials. A comprehensive search of Cochrane Central Register of Controlled Trials was conducted in the Cochrane Library and PubMed, to identify relevant articles focusing on SGLT2i and chronic kidney disease (CKD) in diabetic patients. The most recent article search was conducted on July 12, 2021.

Results: Seven randomized controlled trials (RCTs) were included in the meta-analysis. Two trials were comparing dapagliflozin, two comparing empagliflozin, one comparing ertugliflozin, one comparing canagliflozin, and one comparing sotagliflozin. Composite renal outcome and acute kidney injury (AKI) was found in seven and four studies, respectively. Data on end-stage kidney disease (ESKD) and albuminuria or initiation of renal replacement therapy were reported in the two studies. The pooled risk ratio (RR) $95 \%$ confidence interval (CI) for the composite renal outcome was $0.54(0.50-0.59)$, with $92 \%$ heterogeneity. The pooled RR for AKI was 0.77 (0.66-0.89), with no heterogeneity. A significant lower incidence of albuminuria (RR: 0.69 ; 95\% CI: $0.59-0.81$ ), initiation of renal replacement therapy (RR: 0.71; 95\% CI: 0.58-0.87), was observed following the use of SGLT2 inhibitors.

Conclusion: Our findings confirm that the SGLT2 inhibitors can reduce the risk of albuminuria, AKI and renal replacement therapy in ESKD patients with T2D (type 2 diabetes). These meta-analyses provide substantial evidence supporting the beneficial effect of SGLT2 inhibitors on reducing CKD events in individuals with T2D.
\end{abstract}

Implication for health policy/practice/research/medical education:

Sodium-glucose co-transporter 2 inhibitors (SGLT2i) lower blood glucose by reducing glucose reabsorption. SGLT2i was found to be beneficial in diabetic patients in randomized controlled trials. The current meta-analysis found that SGLT2 inhibitors may reduce the risk of kidney damage in T2D patients..

Please cite this paper as: Jahangiri D, Padhi UN, Kumar Verma HK, Lakkakula BVKS, Valizadeh R, Nasri H. Sodium-glucose co-transporter 2 inhibitors (SGLT2i); as a preventive factor of kidney failure in patients with type 2 diabetes; a meta-analysis of randomized controlled trials. J Renal Inj Prev. 2021; 10(4): e35. doi: 10.34172/jrip.2021.35. 


\section{Introduction}

Diabetes is a global public health problem and one of the top 10 causes of death in most developed countries that pose a high risk of severe vascular complications (1). Diabetic nephropathy (DN) is a major burden among the chronic complications of diabetes, which develops in approximately $30 \%$ of diabetic patients and approaching epidemic proportions globally. Indeed, $\mathrm{DN}$ is the leading cause of chronic kidney disease (CKD) in the United States (2). According to International Diabetes Federation, the comparative prevalence of diabetes in 2007 was $8.0 \%$, which is expected to rise to $7.3 \%$ by 2025 (3).

The natural history of DN includes glomerular hyperfiltration, progressive albuminuria, decreased glomerular filtration rate (GFR) and eventually end-stage renal disease (ESRD). Further, smoking and obesity are known risk factors for DN. Ethnic, familial, and genetic factors also play a significant role in disease progression $(4,5)$. There are two distinct pathways, hemodynamic and non-hemodynamic to be involved in the progression of diabetic kidney disease (6). Although the role of hyperglycemia in the pathophysiology of diabetic complications is not fully understood, it has been linked to an increase in intraglomerular pressure, single nephron GFR, and podocyte damage, which further perpetuates renal dysfunction (7). Recently, a study mentioned that metabolic reprogramming is associated with diabetes, leading to tubulointerstitial inflammation and fibrosis (8).

Treatment with renin-angiotensin-aldosterone system (RAAS) inhibitors prevent the major adverse effect of CKD in people with diabetes, and clinical practice guidelines recommend these drugs for the general approach to the all diabetic individuals with kidney disease or at risk for it $(9,10)$.

The recent approval of sodium/glucose co-transporter 2 inhibitors (SGLT2i) creates new therapeutic options for this high-risk diabetes population. SGLT2i are a novel class of diabetes drugs that lower blood glucose levels by decreasing glucose reabsorption in the proximal renal tubule, resulting in increased urinary glucose and sodium excretion (11,12). Recently, SGLT2i have significantly reduced CKD progression in people with diabetes $(13,14)$. Several clinical trials have found that combining SGLT2 and metformin as an initial treatment for diabetic patients is surprisingly beneficial $(15,16)$. The American Diabetes Association 2020 guidelines recommend prescribing an SGLT2i after a trial of lifestyle modifications in patients with CKD (17).

The benefits of SGLT2i in diabetic patients overcome the moderate side effects observed in the literature. The SGLT2i have been associated with an increased risk of glycosuria and risk for genital mycotic infections (18). However, in diabetes patients with early signs of DN, the specific role of SGLT2i and risk of acute kidney injury (AKI) needs to be defined. Thus, the present meta- analysis aims to identify the effect of SGLT2i on renal pathophysiological events in seven previous published randomized controlled trials (RCTs).

\section{Materials and Methods}

\section{Data sources and search strategy}

The present meta-analysis was conducted in accordance with the PRISMA guideline (19) (Figure 1). Two investigators independently searched Cochrane Central Register of Controlled Trials in the Cochrane Library and PubMed for randomized, placebo-controlled studies involving SGLT2i with endpoints such as impaired renal function, acute kidney injury, and composite renal outcome. The keywords searched were "sodium-glucose co-transporter 2 inhibitors", "Sodium-Glucose Transporter 2 Inhibitors", "SGLT2i", "kidney failure", "type 2 diabetes", "T2D”, "chronic kidney disease", “CKD”, "acute kidney injury", "AKI”, "albuminuria”, "renal replacement therapy”, "RRT”, "hemodialysis", "Sotagliflozin", "peritoneal dialysis", "canagliflozin", “metformin”, "dapagliflozin”, "saxagliptin”, "empagliflozin", "ertugliflozin" or a combination of them in the titles or abstracts. The references of the published articles were manually reviewed for additional relevant articles. The duplicate studies were removed by EndNote. No limitations were set on the language, article timeframe or any other trial characteristics; however, the final literature search was conducted on July 12, 2021.

\section{Inclusion and exclusion criteria}

Our main aim was to assess the effect of SGLT2 inhibitors on DN outcomes; however, after initial screening, the full

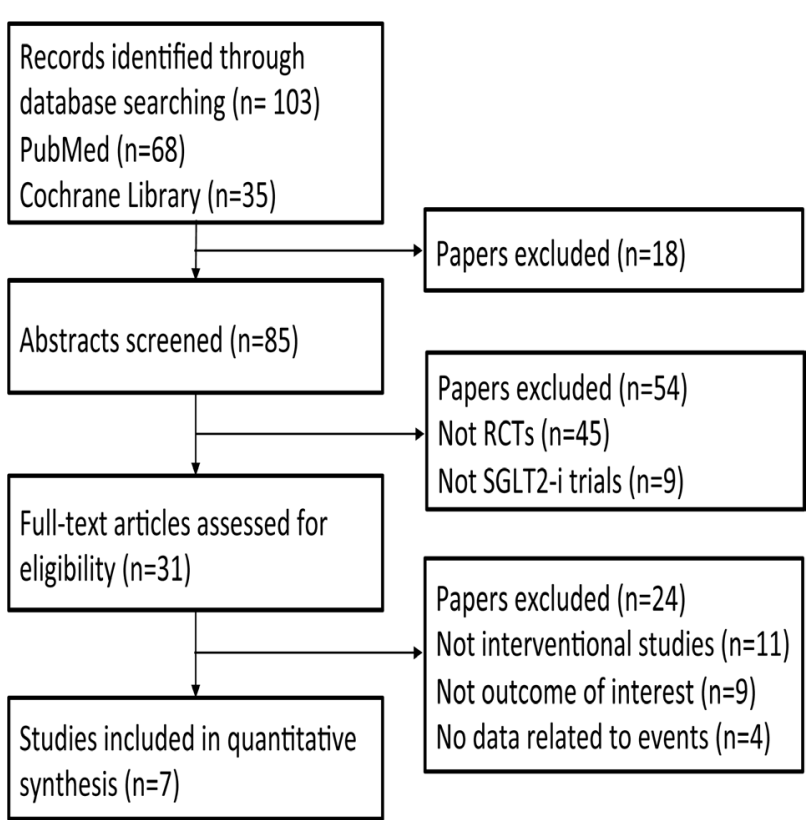

Figure 1. Flow diagram depicting the process of selecting RCTs for inclusion in the meta-analysis. 
text of all relevant papers were obtained and were screened based on inclusion criteria; (1) having placebo/control arm in the design (2) presence of association between renal outcomes and SGLT2i in the study ( 3 ) adults ( $>18$ years) with type 2 diabetes with detailed information preferred. Exclusion criteria were as follows; (1) duplicate studies (2) letters, case reports, editorials, comments, or animal studies (3) trials on people with type 1 diabetes mellitus.

\section{Outcome definitions}

Composite renal outcome is defined as $\geq 40 \%$ reduction in eGFR, the need for renal replacement therapy, doubling serum creatinine or kidney related mortality. AKI is determined by the KDIGO (Kidney Disease: Improving Global Outcomes available at https://web.archive.org/ web/20160304025332/http://www.kdigo.org/clinical_ practice_guidelines/pdf/CKD/KDIGO_2012_CKD_ GL.pdf) definition. Albuminuria was defined by urine albumin: creatinine ratio (ACR) (moderate: $30-300 \mathrm{mg} / \mathrm{g}$ or heavy: $>300 \mathrm{mg} / \mathrm{g}$ ). Serum creatinine criteria (increase in serum creatinine by $\geq 0.3 \mathrm{mg} / \mathrm{dL}$ within $48 \mathrm{~h}$ or increase in serum creatinine by $\geq 1.5$ times baseline value in the prior 7 days). End-stage kidney disease (ESKD) is defined as $\mathrm{eGFR} \leq 30 \mathrm{~mL} / \mathrm{min} / 1.73 \mathrm{~m}^{2}$.

\section{Data collection}

The renal outcomes studied in this meta-analysis include composite renal outcomes [doubling of serum creatinine or $50 \%$ reduction in eGFR (estimated glomerular filtration rate)], AKI, albuminuria, or initiation of renal replacement therapy. Two authors separately collected all data, including date of publication, number of renal outcome events in SGLT2i and placebo groups. The disagreements between the investigators were resolved through discussion with the independent supervisor.

\section{Statistical analysis}

Cochrane Collaboration's risk-of-bias tool was used to assess selection, performance, detection, attrition and reporting biases of different trials (20). Heterogeneity was assessed using Cochran's $\mathrm{Q}$ test and $\mathrm{I}^{2}$ statistics and the value with $\mathrm{I}^{2}>50 \%$ indicated significance (21). Pooled relative risk (RR) and their $95 \%$ confidence interval (CI) were calculated in a fixed-effects model using RevMan version 5.3. Begg's funnel plots were used for assessing publication bias.

\section{Results}

Study selection process

The online search strategy retrieved 103 papers from PubMed $(n=68)$ and Cochrane Library $(n=35)$. Eighteen papers were excluded due to duplication based on the inclusion and exclusion criteria. Following the review of the title and abstract, 54 papers were ruled out. The full article was obtained for the remaining 31 papers. Furthermore, 24 articles were omitted because they neither deal with outcome nor events. Seven RCTs were finally included in the meta-analysis. The study selection process and reasons for exclusions were described in Figure 1. The last updated search was conducted in July 2021.

\section{Study characteristics}

Finally, seven RCTs were selected for this meta-analysis. A total of 55265 individuals were identified (30097 in the SGLT2i group and 25168 in the control group). There were 2 trials comparing empagliflozin versus placebo $(22,23)$; 2 trials comparing dapagliflozin versus placebo $(24,25) ; 1$ trial comparing canagliflozin versus placebo (26); 1 trial comparing ertugliflozin versus placebo (27); 1 trial comparing sotagliflozin versus placebo (28). Characteristics of eligible studies are shown in Table 1. The lower risk of bias indicated that the studies included in this meta-analysis were well-designed and conducted.

\section{Effect of SGLT2i on renal outcomes}

Data on composite renal outcomes were available for 55265 participants (30 097 in the SGLT2i group and 25168 in the control group) in 7 studies (22-28). AKI was reported in 4 studies with 20950 patients in the SGLT2i group and 15844 patients in the placebo group (22,25-27). Data on albuminuria or initiation of renal replacement therapy was reported in 4 studies with 4354 patients in the SGLT2i group and 4351 patients in the placebo group $(24,26)$. Table 2 summarizes the pooled RR with $95 \%$ CI for the renal outcomes. The pooled RR for the composite renal outcome was 0.54 (95\% CI: 0.50-0.59), with heterogeneity of $92 \%$, while the pooled RR for the AKI was 0.77 (95\% CI: $0.66-0.89$ ) with $0 \%$ heterogeneity (Figures $2 \mathrm{~A}$ and $2 \mathrm{~B}$ ). A significant lower incidence of albuminuria (RR: 0.69; 95\% CI: 0.59-0.81), initiation of renal replacement therapy (RR: 0.71 ; 95\% CI: 0.58-0.87), was observed without significant heterogeneity (Figures $2 \mathrm{C}$ and $2 \mathrm{D}$ ).

\section{Publication bias}

Publication bias statistics determined by the Begg's funnel plot indicated that there is no significant asymmetry in funnel plots for the composite renal outcome and AKI (Figure 3A and 3B). These plots visually indicate that there is no publication bias, due to less number studies included in the meta-analysis. As the coefficients are dependent on number of studies, checking the plots visually is of great importance. We could not conduct a test for publication bias for the remaining outcomes due to less number of studies.

\section{Discussion}

The current meta-analysis summarized data on various renal outcomes from seven RCTs involving a range of SGLT2i. The pooled RR indicated that the SGLT2i reduced the incidence of various renal outcomes. Further, Begg's funnel plots demonstrate that there is obvious publication bias. SGLT2 inhibitors protect patients with 
Table 1. Characteristics of studies included in the meta-analysis

\begin{tabular}{|c|c|c|c|c|c|c|c|c|c|c|c|}
\hline Study & Clinical trial name & Clinical trial No. & Drug & SGLT2i & Placebo & $\begin{array}{c}\text { Age } \\
\text { Mean } \pm S D\end{array}$ & Diabetic (\%) & Women (\%) & $\begin{array}{c}\text { eGFR }<60 \mathrm{ml} / \mathrm{min} / 1.73 \mathrm{~m} 2 \\
\% \text { at baseline }\end{array}$ & HbA1c \% baseline & $\begin{array}{l}\text { Follow-up time } \\
\text { (Median years) }\end{array}$ \\
\hline Zinman et al (22) & EMPA-REG OUTCOME & NCT01131676 & Empagliflozin & 4687 & 2333 & $63 \pm 9$ & 100 & 29 & 25.9 & $8.1 \pm 0.8$ & 3.1 \\
\hline Packer et al (23) & EMPEROR-REDUCED & NCT03057977 & Empagliflozin & 1683 & 1867 & $67 \pm 11$ & 50 & 24 & 48 & NA & 1.3 \\
\hline Cannon et al (27) & VERTIS-CV & NCT01986881 & Ertugliflozin & 5499 & 2747 & $64 \pm 8$ & 100 & 30 & 21.9 & $8.2 \pm 1$ & 3 \\
\hline Bhatt et al (28) & SCORED & NCT03315143 & Sotagliflozin & 5292 & 5292 & $68.66 \pm 8$ & 100 & 44.3 & 100 & $8.3 \pm 0.9$ & 1.4 \\
\hline Heerspink et al (24) & DAPA-CKD & NСT03036150 & Dapagliflozin & 2152 & 2152 & $61.8 \pm 12$ & 68 & 33 & 89.1 & NA & 2.4 \\
\hline Wiviott et al (25) & DECLARE-TIMI 58 & NCT01730534 & Dapagliflozin & 8582 & 8578 & $64 \pm 7$ & 100 & 37 & 7.4 & $8.3 \pm 1.2$ & 4.2 \\
\hline Perkovic et al (26) & CREDENCE & NCT02065791 & Canagliflozin & 2202 & 2199 & $63 \pm 9$ & 100 & 34 & 59.8 & $8.3 \pm 1.3$ & 2.6 \\
\hline
\end{tabular}

Table 2. Meta-analysis of SGLT2i according to various kidney related outcomes

\begin{tabular}{|c|c|c|c|c|c|c|c|}
\hline \multirow{2}{*}{ Outcome } & \multirow{2}{*}{ Number of studies } & \multirow{2}{*}{ Number of Participants } & \multicolumn{2}{|c|}{ Heterogeneity } & \multirow{2}{*}{ Risk ratio $(95 \% \mathrm{Cl})$} & \multicolumn{2}{|c|}{ Pooled effect } \\
\hline & & & $12 \%$ & $P$ value & & Z-value & $P$ value \\
\hline Composite renal Outcome & 7 & 55265 & $92 \%$ & $<0.001$ & $0.54(0.50-0.59)$ & 13.62 & $<0.001$ \\
\hline Acute kidney injury & 4 & 36798 & $0 \%$ & 0.44 & $0.77(066-0.89)$ & 3.60 & $<0.001$ \\
\hline Albuminuria & 2 & 8705 & $0 \%$ & 0.83 & $0.69(0.59-0.81)$ & 4.42 & $<0.001$ \\
\hline Initiation of renal replacement therapy & 2 & 8705 & $0 \%$ & 0.53 & $0.71(0.58-0.87)$ & 3.24 & 0.001 \\
\hline
\end{tabular}




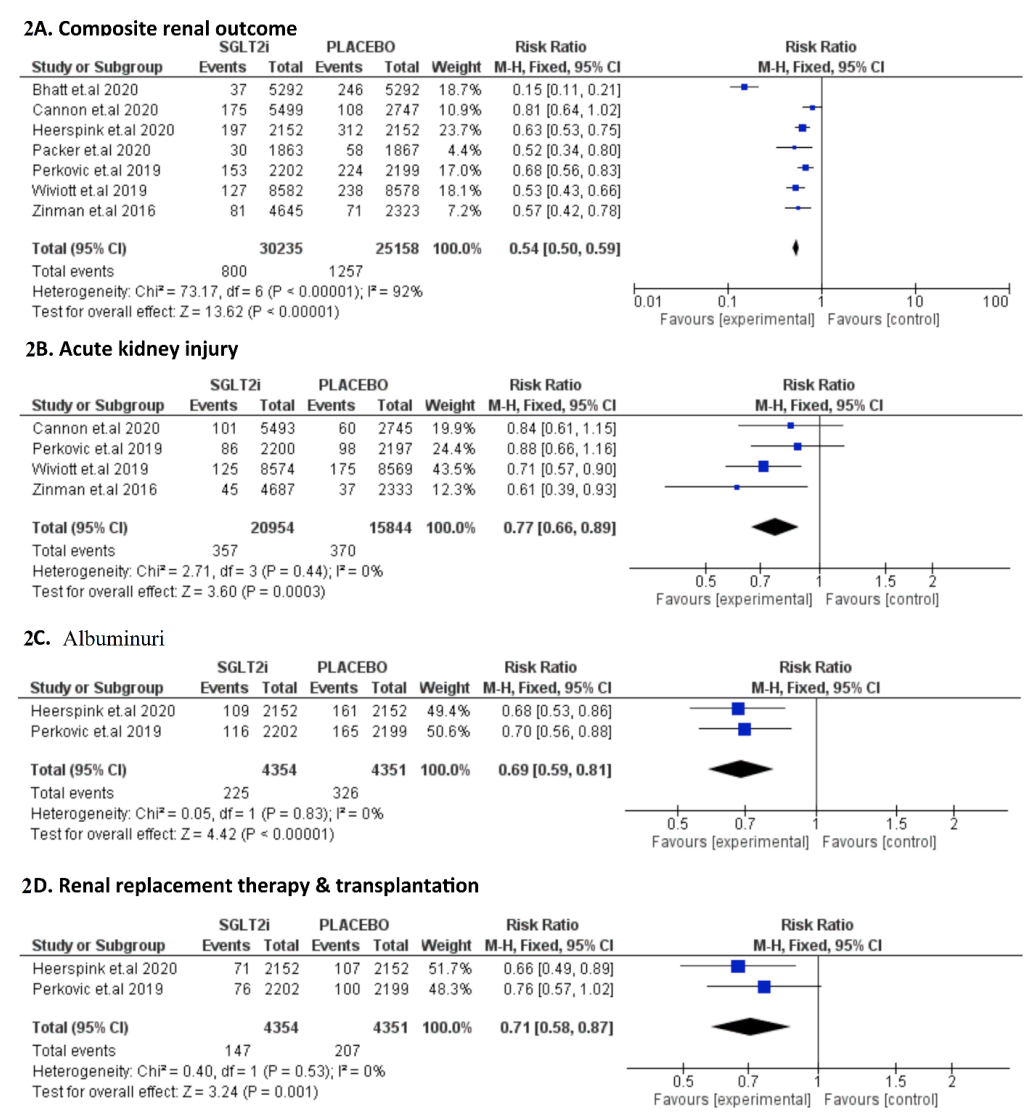

Figure 2. Forest plots illustrating the pooled analysis for renal outcome

diabetes and CKD via two distinct mechanisms. First, in diabetic patients, up-regulation of SGLT2 increases sodium and glucose reabsorption by the proximal tubules and lowers blood sugar by inhibition of SGLT2 glucose reabsorption in the renal proximal tubules. Second, SGLT2i inhibit glucose and sodium reabsorption in the proximal tubules while increasing sodium transport to the macula densa, restoring impaired tubuloglomerular feedback. This demonstrates the impact of SGLT2i on renal hemodynamics. This study improves understanding of essential differences in outcomes related to drugs within the class.

Most randomized evidence supporting positive effect of metformin on patient-level outcomes, demonstrating that metformin reduces the risk of major adverse outcomes, including cardiovascular and renal outcomes, when compared to other early glucose-lowering medications (29). However, metformin alone could not achieve adequate glucose control and metformin neither prevents nor delays complications and maintain quality of life. Several lines of evidences indicate that the SGLT2i were associated with a significantly lower risk of development or progression of ESRD. Despite the significant data of clinical benefits of SGLT2 inhibitor, some guidelines recommend them as the preferred second-line therapy in people with concomitant CKD (30). To date, RCTs evaluating the safety and effectiveness of SGLT2i in patients with T2D have primarily focused on kidney disease outcomes. In this context, it is important to note that SGLT2i was found to be beneficial in trials for dapagliflozin, ertugliflozin, empagliflozin, sotagliflozin, and canagliflozin (22-28). Besides, empagliflozin reduced the urinary albumin to creatinine ratio (UACR) in patients with $\mathrm{T} 2 \mathrm{D}$ regardless of hemoglobin $\mathrm{HbA1c}$, blood pressure, and body weight (31). A study also found that dapagliflozin reduced the UACR in stage 3 CKD patients after 2 years of treatment (32). These findings suggested that SGLT2i could reduce albuminuria by affecting the kidney directly through various mechanisms, including decreased glomerular hyperfiltration, improved tubulointerstitial fibrosis, lower systemic blood pressure, changes in plasma volume expansion, and lower uric acid levels (33-35).

In vivo models also demonstrated that SGLT2 $i$ reduced albuminuria in diabetic mice by improving intra-glomerular hyperfiltration and tubule-interstitial fibrosis, which are two major contributors to renal damage in diabetic kidney disease $(36,37)$. In agreement with these findings, our meta-analysis revealed the albuminuria-lowering effects of SGLT2i. However, the accumulation of data suggests that SGLT2i may be beneficial for albuminuria in the ESRD rather than the early stage, which needs to be investigated further. The 


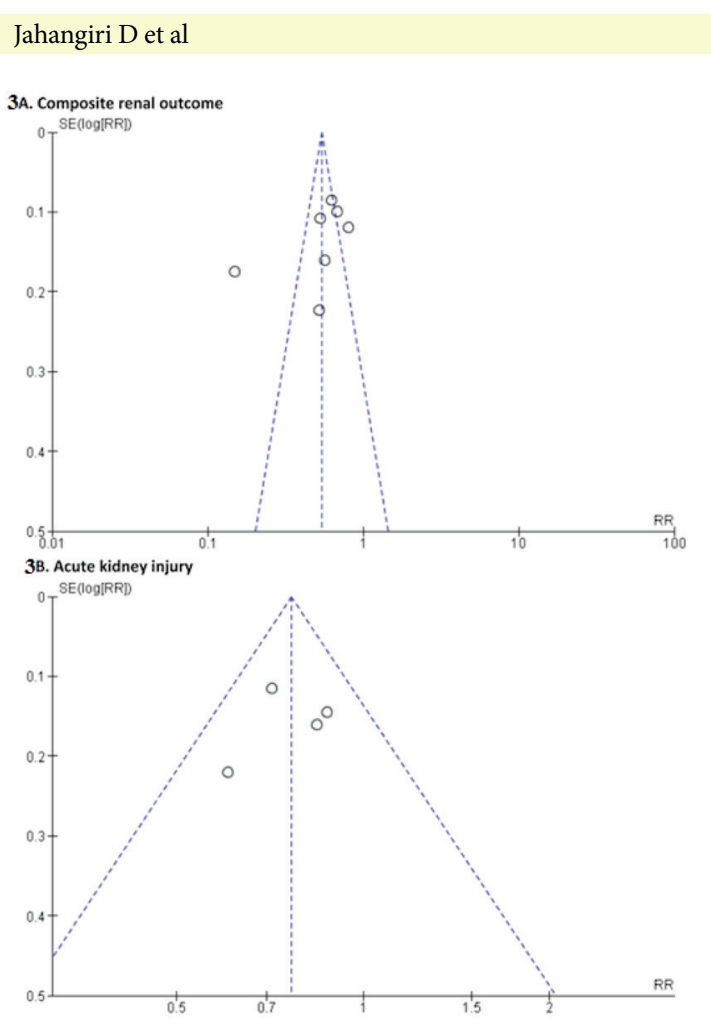

Figure 3. A funnel plot illustrating the publication bias

overall changes in kidney disease showed a difference between SGLT2 inhibitors and controls. In the subgroup analysis, we discovered that the changes in renal outcome after SGLT2i treatment were characterized by a rapid decline in eGFR within the first 4-5 weeks, followed by progressive recovery over time. Furthermore, the eGFR level was reversible within two weeks of drug discontinuation $(38,39)$. The evidence from SGLT2i RCT trials significantly reduced the risk of albuminuria, AKI and renal replacement therapy of ESRD compared with placebo. Protection against AKI is a welcome discovery. These findings support a recently published meta-analysis that found SGLT2i protect against the risk of amputation, fracture, hyperkalemia, hypoglycemia, volume depletion, or UTI (40). Despite this, the large number of events and consistency of effect across trials lends credence to the finding that SGLT2i protect against AKI. Further research is needed to understand the mechanism of decreased AKI risk $(41,42)$.

There are several limitations to this study that should be discussed. First, the analysis included a total of seven RCT studies. Second, the majority of the studies reported incomplete outcome data due to loss to follow-up, which necessitates further investigation. Third, we were unable to assess the renal effects of SGLT2 inhibitors based on CKD risk. In summary, our findings confirm that SGLT2 inhibition can reduce the risk of albuminuria, AKI and renal replacement therapy in ESRD patients with T2D. These meta-analyses provide substantial evidence supporting the beneficial effect of SGLT2 inhibitors on reducing $\mathrm{CKD}$ events in individuals with $\mathrm{T} 2 \mathrm{D}$.

\section{Authors' contribution}

DJ and HN conducted the primary search. UNP participated in methodological search and data collection. HKV conducted the draft. LVKSB conducted the primary revisions. RV conducted the secondary edit. HN and DJ finalized the manuscript. All authors read and signed the final paper.

\section{Conflicts of interest}

The authors declare that there is no potential conflict of interest.

\section{Ethical issues}

The institutional ethical committee of Isfahan University of Medical Sciences approved all study protocols (Ethical code\# IR.MUI.MED.REC.1400.279). This study was extracted from the M.D., thesis of Dorsa Jahangiri at this university (Thesis \# 3400263). Additionally, ethical issues (including plagiarism, data fabrication, double publication) have been completely observed by the authors.

\section{Funding/Support}

None.

\section{References}

1. Lin, X, Xu Y, Pan X, Xu J, Ding Y, Sun X, et al. Global, regional, and national burden and trend of diabetes in 195 countries and territories: an analysis from 1990 to 2025 . Sci Rep. 2020;10:14790. doi: 10.1038/s41598-020-71908-9.

2. Alicic RZ, Rooney MT, Tuttle KR. Diabetic Kidney Disease. Challenges, Progress, and Possibilities. Clin J Am Soc Nephrol. 2017;12:2032-45. doi: 10.2215/cjn.11491116.

3. Saeedi P, Petersohn I, Salpea P, Malanda B, Karuranga S, Unwin N, et al. Global and regional diabetes prevalence estimates for 2019 and projections for 2030 and 2045: Results from the International Diabetes Federation Diabetes Atlas, 9(th) edition. Diabetes Res Clin Pract. 2019;157:107843. doi: 10.1016/j.diabres.2019.107843.

4. Giralt-Lopez A, Molina-Van den Bosch M, Vergara A, Garcia-Carro C, Seron D, Jacobs-Cacha C, et al. Revisiting experimental models of diabetic nephropathy. Int J Mol Sci. 2020;21:3587. doi: 10.3390/ijms21103587.

5. Natesan V, Kim SJ. Diabetic nephropathy - a review of risk factors, progression, mechanism, and dietary management. Biomol Ther (Seoul). 2021;29:365-72. doi: 10.4062/ biomolther.2020.204.

6. Badal SS, Danesh FR. New insights into molecular mechanisms of diabetic kidney disease. Am J Kidney Dis. 2014;63:S63-83. doi: 10.1053/j.ajkd.2013.10.047.

7. Tonneijck L, Muskiet MH, Smits MM, van Bommel EJ, Heerspink HJ, van Raalte DH, et al. Glomerular hyperfiltration in diabetes: mechanisms, clinical significance, and treatment. J Am Soc Nephrol. 2017; 28:1023-39. doi: 10.1681/ASN.2016060666.

8. Zheng W, Guo J, Liu ZS. Effects of metabolic memory 
on inflammation and fibrosis associated with diabetic kidney disease: an epigenetic perspective. Clin Epigenetics. 2021;13:87. doi: 10.1186/s13148-021-01079-5.

9. Mavrakanas TA, Lipman ML. Angiotensin-converting enzyme inhibitors vs. angiotensin receptor blockers for the treatment of hypertension in adults with type 2 diabetes: why we favour angiotensin receptor blockers. Can J Diabetes. 2018;42:118-23. doi: 10.1016/j.jcjd.2017.11.006.

10. Wang $\mathrm{K}, \mathrm{Hu}$ J, Luo T, Wang Y, Yang S, Qing $\mathrm{H}$, et al. Effects of angiotensin-converting enzyme inhibitors and angiotensin II receptor blockers on all-cause mortality and renal outcomes in patients with diabetes and albuminuria: a systematic review and meta-analysis. Kidney Blood Press Res. 2018;43:768-79. doi: 10.1159/000489913.

11. Scheen AJ. Pharmacodynamics, efficacy and safety of sodium-glucose co-transporter type 2 (SGLT2) inhibitors for the treatment of type 2 diabetes mellitus. Drugs. 2015;75:33-59. doi: 10.1007/s40265-014-0337-y.

12. Jung CH, Jang JE, Park J-Y. A novel therapeutic agent for type 2 diabetes mellitus: SGLT2 inhibitor. Diabetes Metab J. 2014;38:261-73. doi: 10.4093/dmj.2014.38.4.261.

13. Wheeler DC, James J, Patel D, Viljoen A, Ali A, Evans M, et al. SGLT2 inhibitors: slowing of chronic kidney disease progression in type 2 diabetes. Diabetes Ther. 2020;11:275774. doi: 10.1007/s13300-020-00930-x.

14. Dekkers CCJ, Gansevoort RT. Sodium-glucose cotransporter 2 inhibitors: extending the indication to nondiabetic kidney disease? Nephrol Dial Transplant. 2020; 35:i33-42. doi: 10.1093/ndt/gfz264.

15. Hadjadj S, Rosenstock J, Meinicke T, Woerle HJ, Broedl UC. Initial combination of empagliflozin and metformin in patients with type 2 diabetes. Diabetes Care. 2016;39:171828. doi: $10.2337 / \mathrm{dc} 16-0522$.

16. Rosenstock J, Chuck L, González-Ortiz M, Merton K, Craig J, Capuano G, et al. Initial combination therapy with canagliflozin plus metformin versus each component as monotherapy for Drug-Naïve type 2 diabetes. Diabetes Care. 2016;39:353-62. doi: 10.2337/dc15-1736.

17. Introduction: Standards of Medical Care in Diabetes-2020. Diabetes Care. 2020;43:S1-S2. doi: 10.2337/dc20-Sint.

18. Singh M, Kumar A. Risks associated with SGLT2 inhibitors: an overview. Curr Drug Saf. 2018;13:84-91. doi: 10.2174/15 74886313666180226103408.

19. Shamseer L, Moher D, Clarke M, Ghersi D, Liberati A, Petticrew M, et al. Preferred reporting items for systematic review and meta-analysis protocols (PRISMA-P) 2015: elaboration and explanation. BMJ. 2015;349:g7647. doi: 10.1136/bmj.g7647.

20. Higgins JP, Savović J, Page MJ, Elbers RG, Sterne JA. Assessing risk of bias in a randomized trial. In: Cochrane Handbook for Systematic Reviews of Interventions. The Cochrane Collaboration; 2019. p. 205-28.

21. Engels EA, Schmid CH, Terrin N, Olkin I, Lau J. Heterogeneity and statistical significance in metaanalysis: an empirical study of 125 meta-analyses. Stat Med. 2000;19:1707-28. doi: https://doi.org/10.1002/10970258(20000715)19:13<1707::AID-SIM491>3.0.CO;2-P.

22. Zinman B, Lachin JM, Inzucchi SE. Empagliflozin, cardiovascular outcomes, and mortality in type 2 Diabetes. N Engl J Med. 2016;374:1094. doi: 10.1056/NEJMc1600827.

23. Packer M, Anker SD, Butler J, Filippatos G, Pocock
SJ, Carson P, et al. Cardiovascular and renal outcomes with empagliflozin in heart failure. N Engl J Med. 2020; 383:1413-24. doi: 10.1056/NEJMoa2022190.

24. Heerspink HJL, Stefánsson BV, Correa-Rotter R, Chertow GM, Greene T, Hou FF, et al. Dapagliflozin in patients with chronic kidney disease. N Engl J Med. 2020;383:1436-46. doi: 10.1056/NEJMoa2024816.

25. Wiviott SD, Raz I, Bonaca MP, Mosenzon O, Kato ET, Cahn A, et al. Dapagliflozin and cardiovascular outcomes in type 2 diabetes. N Engl J Med. 2019;380:347-57. doi: 10.1056/ NEJMoa1812389.

26. Perkovic V, Jardine MJ, Neal B, Bompoint S, Heerspink HJL, Charytan DM, et al. Canagliflozin and renal outcomes in type 2 diabetes and nephropathy. $\mathrm{N}$ Engl J Med. 2019;380:2295-306. doi: 10.1056/NEJMoa1811744.

27. Cannon CP, Pratley R, Dagogo-Jack S, Mancuso J, Huyck S, Masiukiewicz U, et al. Cardiovascular outcomes with ertugliflozin in type 2 diabetes. $\mathrm{N}$ Engl J Med. 2020;383:1425-35. doi: 10.1056/NEJMoa2004967.

28. Bhatt DL, Szarek M, Pitt B, Cannon CP, Leiter LA, McGuire $\mathrm{DK}$, et al. Sotagliflozin in patients with diabetes and chronic kidney disease. N Engl J Med. 2021;384:129-39. doi: 10.1056/NEJMoa2030186.

29. Petrie JR, Rossing PR, Campbell IW. Metformin and cardiorenal outcomes in diabetes: A reappraisal. Diabetes, Obesity and Metabolism. 2020;22:904-15. doi: 10.1111/ dom.13984.

30. Tuttle KR, Brosius FC, Cavender MA, Fioretto P, Fowler KJ, Heerspink HJL, et al. SGLT2 Inhibition for CKD and Cardiovascular Disease in Type 2 Diabetes: Report of a Scientific Workshop Sponsored by the National Kidney Foundation. Diabetes. 2021;70:1-16. doi: 10.2337/dbi200040 .

31. Cherney D, Lund SS, Perkins BA, Groop PH, Cooper ME, Kaspers $\mathrm{S}$, et al. The effect of sodium glucose cotransporter 2 inhibition with empagliflozin on microalbuminuria and macroalbuminuria in patients with type 2 diabetes. Diabetologia. 2016;59:1860-70. doi: 10.1007/s00125-0164008-2.

32. Fioretto P, Zambon A, Rossato M, Busetto L, Vettor R. SGLT2 inhibitors and the diabetic kidney. Diabetes Care. 2016;39:S165-71. doi: 10.2337/dcS15-3006.

33. Cherney DZ, Perkins BA, Soleymanlou N, Maione M, Lai V, Lee A, et al. Renal hemodynamic effect of sodium-glucose cotransporter 2 inhibition in patients with type 1 diabetes mellitus. Circulation. 2014;129:587-97. doi: 10.1161/ CIRCULATIONAHA.113.005081.

34. Panchapakesan U, Pegg K, Gross S, Komala MG, Mudaliar $\mathrm{H}$, Forbes J, et al. Effects of SGLT2 inhibition in human kidney proximal tubular cells--renoprotection in diabetic nephropathy? PLoS One. 2013;8:e54442. doi: 10.1371/ journal.pone.0054442.

35. Lambers Heerspink HJ, de Zeeuw D, Wie L, Leslie B, List J. Dapagliflozin a glucose-regulating drug with diuretic properties in subjects with type 2 diabetes. Diabetes Obes Metab. 2013;15:853-62. doi: 10.1111/dom.12127.

36. Gembardt F, Bartaun C, Jarzebska N, Mayoux E, Todorov VT, Hohenstein B, et al. The SGLT2 inhibitor empagliflozin ameliorates early features of diabetic nephropathy in BTBR ob/ob type 2 diabetic mice with and without hypertension. Am J Physiol Renal Physiol. 2014;307:F317-25. doi: 
10.1152/ajprenal.00145.2014.

37. Terami N, Ogawa D, Tachibana H, Hatanaka T, Wada J, Nakatsuka A, et al. Long-term treatment with the sodium glucose cotransporter 2 inhibitor, dapagliflozin, ameliorates glucose homeostasis and diabetic nephropathy in $\mathrm{db} / \mathrm{db}$ mice. PLoS One. 2014;9:e100777. doi: 10.1371/journal. pone. 0100777 .

38. Kohan DE, Fioretto P, Tang W, List JF. Long-term study of patients with type 2 diabetes and moderate renal impairment shows that dapagliflozin reduces weight and blood pressure but does not improve glycemic control. Kidney Int. 2014;85:962-71. doi: 10.1038/ki.2013.356.

39. Barnett AH, Mithal A, Manassie J, Jones R, Rattunde H, Woerle HJ, et al. Efficacy and safety of empagliflozin added to existing antidiabetes treatment in patients with type 2 diabetes and chronic kidney disease: a randomised, double- blind, placebo-controlled trial. Lancet Diabetes Endocrinol. 2014;2:369-84. doi: 10.1016/S2213-8587(13)70208-0.

40. Bai Y, Jin J, Zhou W, Zhang S, Xu J. The safety outcomes of sodium-glucose cotransporter 2 inhibitors in patients with different renal function: A systematic review and meta-analysis. Nutrition, Metabolism and Cardiovascular Diseases. 2021;31:1365-74. doi: 10.1016/j. numecd.2021.02.006.

41. Heerspink HJL, Kosiborod M, Inzucchi SE, Cherney DZI. Renoprotective effects of sodium-glucose cotransporter-2 inhibitors. Kidney Int. 2018;94:26-39. doi: 10.1016/j. kint.2017.12.027.

42. Chang YK, Choi H, Jeong JY, Na KR, Lee KW, Lim BJ, et al. Dapagliflozin, SGLT2 inhibitor, attenuates renal ischemiareperfusion injury. PLoS One. 2016;11:e0158810. doi: 10.1371/journal.pone.0158810.

Copyright $\odot 2021$ The Author(s); Published by Nickan Research Institute. This is an open-access article distributed under the terms of the Creative Commons Attribution License (http://creativecommons.org/licenses/by/4.0), which permits unrestricted use, distribution, and reproduction in any medium, provided the original work is properly cited. 